\title{
Genomic Analyses of Cherry Rusty Mottle Group and Cherry Twisted Leaf-Associated Viruses Reveal a Possible New Genus Within the Family Betaflexiviridae
}

\author{
D. E. V. Villamor, J. Susaimuthu, and K. C. Eastwell
}

Department of Plant Pathology, Washington State University-Irrigated Agriculture Research and Extension Center, Prosser 99350. Accepted for publication 12 September 2014.

\begin{abstract}
Villamor, D. E. V., Susaimuthu, J., and Eastwell, K. C. 2015. Genomic analyses of cherry rusty mottle group and cherry twisted leaf-associated viruses reveal a possible new genus within the family Betaflexiviridae. Phytopathology 105:399-408.

It is demonstrated that closely related viruses within the family Betaflexiviridae are associated with a number of diseases that affect sweet cherry (Prunus avium) and other Prunus spp. Cherry rusty mottleassociated virus $(\mathrm{CRMaV})$ is correlated with the appearance of cherry rusty mottle disease (CRMD), and Cherry twisted leaf-associated virus (CTLaV) is linked to cherry twisted leaf disease (CTLD) and apricot ringpox disease (ARPD). Comprehensive analysis of previously reported

full genomic sequences plus those determined in this study representing isolates of CTLaV, CRMaV, Cherry green ring mottle virus, and Cherry necrotic rusty mottle virus revealed segregation of sequences into four clades corresponding to distinct virus species. High-throughput sequencing of RNA from representative source trees for CRMD, CTLD, and ARPD did not reveal additional unique virus sequences that might be associated with these diseases, thereby further substantiating the association of CRMaV and CTLaV with CRMD and CTLD or ARPD, respectively. Based on comparison of the nucleotide and amino acid sequence identity values, phylogenetic relationships with other triple-gene blockcoding viruses within the family Betaflexiviridae, genome organization, and natural host range, a new genus (Robigovirus) is suggested.
\end{abstract}

A number of diseases of sweet cherry (Prunus avium) that were first described in the 1940s $(28,37-39)$ are presumed to have a viral etiology based on the graft-transmissible nature of these diseases. To facilitate disease diagnosis and studies of epidemiology, the nature of the agents is now established.

The rusty mottle group is a complex assemblage of diseases affecting sweet cherry that include cherry rusty mottle disease (CRMD), cherry necrotic rusty mottle disease (CNRMD), Frogmore canker, cherry bark blister, and Lambert mottle. Trees affected with CRMD exhibit chlorotic mottling of basal leaves that abscise prematurely while the remaining leaves become bright yellow or red as the season progresses. In contrast, CNRMDaffected trees of some sweet cherry varieties show distinct angular necrotic leaf spots that turn to shot holes later in the season. Leaf chlorotic mottle symptoms in CNRMD-affected trees appear only later in the season (50). Based on reciprocal graft-transmission studies, the viral agents of cherry bark blister (43), Frogmore virus canker (36), and Lambert mottle (27) diseases are presumed to be identical or closely related to agents that cause CNRMD. Virus-like sequences have recently been reported to be associated with CRMD and CNRMD; they were designated Cherry rusty mottle-associated virus (CRMaV) (48) and Cherry necrotic rusty mottle virus $(25,40)$, respectively.

Cherry twisted leaf disease (CTLD) is characterized by abrupt kinking of the leaf petiole and midrib that progresses to leaf twisting; affected trees are also generally stunted (20). A high molecular weight double-stranded RNA (dsRNA) (19,55) and

Corresponding author: K. C. Eastwell; E-mail address: keastwell@wsu.edu

*The $\boldsymbol{e}$-Xtra logo stands for "electronic extra" and indicates that the online version contains four supplemental tables.

http://dx.doi.org/10.1094/PHYTO-03-14-0066-R

(c) 2015 The American Phytopathological Society flexuous clostero-like virus (21) have been associated with CTLD. Apricot ringpox disease (ARPD) is typified by the appearance of chlorotic rings and ragged holes in the leaves, and discolored rings and spots on the fruits of some apricot cultivars (18). Based on reciprocal graft transmission studies, the causal agent of CTLD is presumed to be closely related to the agent responsible for ARPD $(18,28)$. Whether these two diseases are caused by the same virus has not been firmly established because not all CTLD isolates from cherry induced ARPD in apricot and vice versa (28). Cherry green ring mottle virus (CGRMV) (26) was reported in an apricot showing symptoms similar to those of ARPD. Concurrent to preparation of this manuscript, the first sequence of Cherry twisted leaf-associated virus (CTLaV) was published (22). Notwithstanding the presumed similarity between the causal agents of CTLD and ARPD, no virus sequence has been specifically associated with ARPD.

Analysis of partial genome virus-like sequences reported for CGRMV, CTLaV, CRMaV, and Cherry necrotic rusty mottle virus (CNRMV) revealed a relationship to the sequence of CGRMV $(14,51,54)$. More extensive phylogenetic analyses of the nucleotide sequence of the putative coat protein (CP) genes of several isolates of CRMaV, CTLaV, CNRMV, and CGRMV revealed four distinct clades, each corresponding to individual virus species that correlated strongly with distinct symptom expression on Prunus indicators (46). This analysis also indicated that the CTLaV clade comprises both CTLD- and ARPD-associated virus sequences, thereby supporting the previous assertion that agents causing CTLD and ARPD are closely related $(18,28)$. The taxonomic status of the viruses associated with the diseases remains as unassigned members within the family Betaflexiviridae. The growing number of unassigned viruses placed within the family Betaflexiviridae necessitates a phylogenetic and taxonomic review of this family. Because the number of genomic sequences of CRMaV and CTLaV is currently limited in public databases, additional isolates of these viruses were characterized. 
Advances in sequencing technologies-namely, high-throughput sequencing (next-generation or deep sequencing)-has contributed tremendously to unraveling the nature of microbial agents associated with disease $(9,24,33,45)$. Previously uncharacterized viruses associated with plant diseases of unknown etiology $(1,5,7,8,13,15,35,42,49)$, as well as mycoviruses in a single plant (6), have been enumerated by this technology. In this regard, it is possible that novel viruses, in addition to the previously noted CRMaV or CTLaV, may be revealed by high-throughput sequencing and, ultimately, associated with CRMD and CTLD or ARPD, respectively.

In this study, the full genome sequences of several isolates of CRMaV and CTLaV plus representative isolates of CNRMV and CGRMV were determined. The genome organizations, phylogenetic relationships from different open reading frames (ORFs), and nucleotide and amino acid identity values reveal a possible grouping of these viruses into a single genus within the family Betaflexiviridae. High-throughput sequence analysis of total RNA from representative trees affected by CTLD, ARPD, and CRMD did not reveal any novel virus that might be associated with these diseases other than those previously revealed by reverse-transcription polymerase chain reaction (RT-PCR). This further substantiates the association of CRMaV and CTLaV with CRMD and CTLD or ARPD, respectively.

\section{MATERIALS AND METHODS}

Selection of disease source trees for full genome sequencing. In total, 12 known source trees for CTLaV, CRMaV, CNRMV, and CGRMV were selected for sequencing of the complete genome for each virus following long-range RT-PCR and cloning of the amplicons. Of these, four were CTLD sources (103-15, 101-13, 8265, and 8242-3), two were ARPD sources (95CI205R1 and 95CI206), four were CRMD sources (98CI194, 98CI73R1, 8241-2, and 8099-5), and there was one source each of CNRMD (04E36) and CGRMD (02F23rD). The complete genomes of additional isolates of CTLaV and CRMaV, obtained from source trees ARPD C3 and CRMD 8804, respectively, were determined solely by high-throughput sequencing. All CTLD, CRMD, CGRMD, and CNRMD isolates are maintained in P. avium 'Bing' whereas the two ARPD isolates (95CI205R1 and 95CI206) are maintained in P. armeniaca 'Tilton'; all infected trees were maintained in the screenhouse collection of the Clean Plant Center Northwest (CPCNW) at Washington State University (WSU), Prosser. Budwood from ARPD source tree C3 was obtained from the Canadian Plant Virus Collection, Agriculture and Agri-Food Canada, Summerland, BC, Canada, where it is maintained in $P$. armeniaca 'Hungarian'.

Virological status of CRMD, CTLD, and ARPD source trees. The original CTLD source trees 103-15 and 8242-3, ARPD source tree 95CI205R1, and CRMD source trees 8804 and 95CI192R3 were no longer available at the time of initiation of the high-throughput sequencing study; therefore, trees previously propagated from these sources were used: namely, CTLD 103-15P1 and 8242-3-Bi; ARPD 95CI205R1-P2' and CRMD 8804-2, and 95CI192R3-P2. The virus profiles of these trees were also determined by RT-PCR, as described previously (48), plus additional RT-PCR assays for Cherry virus $A$ (CVA) using a more broad-spectrum primer (30), and specific RT-PCR tests for CTLaV, CNRMV, CRMaV, and CGRMV (46), with supplementary enzyme-linked immunosorbent assay (ELISA) tests for Prune dwarf virus (PDV) (antibody source CPCNW, WSU, Prosser), Prunus necrotic ringspot virus (PNRSV) (AC Diagnostics, Fayetteville, AR), and Cherry leafroll virus (CLRV) (Bioreba, Longmont, CO).

Determination of the $5^{\prime}$ terminus of the virus genome. The sequences of the $5^{\prime}$ terminus of CRMaV isolate 98CI194 and CTLaV isolates 103-15 and 101-13 were determined using a commercial kit for $5^{\prime}$ rapid amplification of cDNA ends (5'RACE) (GeneRacer; Life Technologies, Carlsbad, CA). Primers NGRM 6161R and NGRM 2996R were used for first-strand cDNA synthesis and 5'-RACE, respectively, as described previously (48). Four to five clones of the approximately $3-\mathrm{kb}$ amplification products from each source were partially sequenced (singlepass sequencing with $\mathrm{M} 13 \mathrm{~F}$ and M13R primers) in order to obtain the $5^{\prime}$-terminal sequences.

Sequence analysis of full genomic sequences of CTLaV, CRMaV, CNRMV, and CGRMV. The design of $5^{\prime}$-terminus primers for the amplification of $\mathrm{CRMaV}$ was based on two previously sequenced isolates, 95CI192R3 and B48-C (48), and isolate 98CI194 sequenced in this study. The corresponding $5^{\prime}$-terminus primers for CTLaV were based on the sequences generated by $5^{\prime}$ RACE in this study. For the amplification of CNRMV and CGRMV, 5'-terminus primers were designed from GenBank sequences of three and four isolates, respectively. The 3 '-terminus primers were designed from Fovea2-AdPr virus sequences of individual virus isolates generated in a previous study (46). Fulllength genome RT-PCR amplification of each virus isolate using specific primers (Supplemental Table 1), and cloning and sequencing were carried out as previously described (48). Two to three different clones from a single RT-PCR reaction were selected for sequencing by primer walking.

Assembly of contigs and pairwise alignment of sequences were accomplished with BioEdit software (17). Multiple sequence alignments of the CP amino acid sequences were performed with MUSCLE (12), and phylogenetic analyses of ORFs were carried out using the maximum-likelihood method, applying the JonesTaylor-Thornton (JTT) matrix and partial gap deletion option with 1,000 bootstrap replicates of MEGA program package, version 6 (44).

In addition to disease sources CTLD 103-15-P1 and 8242-3-Bi, ARPD 95CI205R1-P2, and CRMD 8804-2 and 95CI192R3-P2, disease sources CRMD 989CI194, ARPD C3, and 95CI206 were included for high-throughput sequencing. Total RNA was extracted as previously described (48) from dormant buds, and at least $1.5 \mu \mathrm{g}$ of RNA per sample was submitted for library construction using Illumina TruSeq Stranded Total RNA Sample Preparation kit with Plant Ribo-Zero and 50-bp single-end read next-generation sequencing on the Illumina HiSeq 2000 platform with multiplexing (Microarray and Genomic Analysis Core Facility, Huntsman Cancer Institute, University of Utah, Salt Lake City). Removal of adapters and barcode sequences was done at the Microarray and Genomic Analysis Core Facility, and subsequent trimming based on quality scores was done using the CLC Genomics Workbench 5.1 (CLC Bio, Aarhus, Denmark). Sequence reads corresponding to host sequences were first removed using the read mapping software of CLC Genomics workbench (default short-read mapping parameters: mismatch cost $=2$ and limit $=8$ ). The default short-read mapping parameter values allow for two mismatches to the reference sequence plus two unassembled nucleotides at the $5^{\prime}$ or $3^{\prime}$ ends of each read. This translates to a minimum of $92 \%$ nucleotide (nt) identity to the reference sequence before a 50-bp read is included in the mapping. Because of the limited number of $P$. avium or $P$. armeniaca sequences currently available in public databases, sequences specific to $P$. persica, a close relative to either $P$. avium or $P$. armeniaca and whose full genome is already determined, was also used in host sequence reduction. The de novo assembly program of CLC Genomics Workbench was used to assemble the resulting reads into 120-bp contigs. After individual reads were reconfirmed against the assembled contigs, a total of less than 50 contigs had their length reduced to less than $120 \mathrm{bp}$ in length. The contigs were subsequently compared to the nucleotide collection database (nr/nt) of the National Center for Biotechnology Information (NCBI) using BLASTN and BLASTX analyses and employing a $10^{-3}$ e-value cut-off. 
In addition to de novo contig assembly, sequence reads that did not assemble into contigs were mapped to a database of virus and viroid reference sequences obtained from the GenBank. Finally, trimmed sequence reads from each source tree were also mapped to the CTLaV, CRMaV, CGRMV, and CNRMV full genome sequences determined in this study, including corresponding full genome sequences that are available in the GenBank, using the default short-read mapping parameters of CLC Genomics Workbench.

\section{RESULTS}

Virological status of CRMD, CTLD, and ARPD source trees. The virus status of trees selected for analysis was determined by serological and molecular assays (Table 1). None of the disease sources used in this study harbored single virus infections, indicating the difficulty in establishing cause-and-effect relationships. However, in each case, a representative of a specific virus sequence clade was associated with each individual disease. The results of RT-PCR analysis and serological assays were supported by sequence analysis from high-throughput sequencing of select CRMD, CTLD, and ARPD source trees.

Comparison of diagnostic testing methods revealed few inconsistencies (Table 1). Two of the trees used in this study (CRMD 98CI73R1 and CTLD 8265) yielded positive RT-PCR test results for CVA using a recently modified primer (30). However, an assay using previously described primers for CVA (46) failed to detect these two isolates. PDV and PNRSV were not detected in CRMD 95CI192R3-P2 whereas the original source tree, CRMD 95CI192R3, was infected by both viruses (46). In like manner, CGRMV was not detected in ARPD 95CI205R1-P2 but was present in the original source tree, ARPD 95CI205R1 (46). The differences between the virus profiles in these source trees can be explained by the failure of these viruses to be transmitted after graft inoculation. In all cases, the presence of CVA and absence of PDV, PNRSV, and CGRMV in the corresponding source trees were confirmed by the high-throughput sequence analysis (Table 1).

$5^{\prime}$ Terminus of virus genomes. The sequence of the first $500 \mathrm{nt}$ from four clones derived from 5'-RACE of RNA isolated from CRMD 98CI194 shared $>99 \%$ nt identities with each other, and the consensus sequence is designated CRMaV 98CI194. This sequence showed nucleotide identity of $86 \%$ with the corresponding sequences of CRMaV from sources 95CI192R3 and B48-C (48).

Five clones from the 5'-RACE of CTLD 101-13 generated two distinct 500-nt sequences. Two clones had nucleotide identities of 90, 99, 90, and 97\% with CGRMV N, CGRMV P1C124, CGRMV F9, and CGRMV Hs10, respectively. The recovery of CGRMV sequence from CTLD 101-13 was not surprising because this tree was previously shown to be infected with CGRMV (46).

Three 5'-terminus sequences of CTLD 101-13 exhibited $100 \%$ nt identity to each other but only $75 \%$ nt identity to the CGRMV sequences from the same source. These sequences from CTLD 101-13 and the consensus sequence from CTLD 103-15 5 -RACE clones showed high nucleotide identity values with each other $(>99 \%)$ but lower nucleotide identity values with known isolates of CNRMV (75 to 78\%), CGRMV (75 to 76\%), and CRMaV (77 to $79 \%$ ). The consensus sequences obtained after 5 -RACE and cloning were considered to represent the putative $\mathrm{CTLaV}$ sequence from each of these disease isolate.

Sequence analysis of full-length virus genomic clones. The complete nucleotide sequence of the genome of each pertinent virus from individual source trees ranged from 8,372 to 8,434 nt (Supplemental Table 2). The genome organizations of all CRMaV, CTLaV, CNRMV, and CGRMV isolates sequenced in this study are similar to the recently sequenced CRMaV isolates 95CI192R3 and B48-C (48), CTLaV isolate ZH (22), and the CNRMV and CGRMV isolates available in GenBank. The genomes consist of five ORFs plus two additional ORFs, $2 \mathrm{a}$ and $5 \mathrm{a}$, nested within ORF2 and ORF5, respectively.

The lengths of the $5^{\prime}$-untranslated region (UTR) of each virus sequences are identical whereas the length of the $3^{\prime}$-UTR varies slightly depending on virus isolate. ORF1 encodes a putative replicase of 2,026 to 2,029,2,037 to 2,039, 2,038, and 2,026 amino acids (aa) for CRMaV, CTLaV, CNRMV, and CGRMV, respectively. The calculated relative molecular mass (Mr) of this large replicase is approximately $230 \mathrm{kDa}$ and consists of the viral methyltransferase (Met), helicase (Hel), RNA-dependent RNA polymerase (RdRp), AlkB homologue, and ovarian tumor (OTU)like cysteine protease domains present in several members within the family Betaflexiviridae (29,31). Conserved residues and motifs

TABLE 1. Viruses detected in disease source trees used in this study

\begin{tabular}{|c|c|c|c|}
\hline Source tree & Viruses found $^{a}$ & High-throughput sequencing & Citation \\
\hline CRMD 8099-5 & CRMaV, CVA, PDV & No & 46 \\
\hline CRMD 8241-2 & CRMaV, CMLV, CVA, PDV & No & 46 \\
\hline CRMD 98CI73R1 & CRMaV, CVA, PDV & No & 46; This studyb \\
\hline CRMD 95CI192R3-P2 ${ }^{\mathrm{c}}$ & CRMaV, CVA & Yes & This study \\
\hline CRMD 8804 & $\mathrm{CRMaV}$ & No & 48 \\
\hline CRMD $8804-2^{\mathrm{c}}$ & CRMaV, CVA & Yes & This study \\
\hline CRMD 98CI194 & CRMaV, CVA & Yes & 46 \\
\hline CTLD 103-15 & ACLSV, CGRMV, CMLV, CTLaV & No & 46 \\
\hline CTLD $103-15-\mathrm{P} 1^{\mathrm{c}}$ & ACLSV, CGRMV, CMLV, CTLaV, CVA & Yes & This study \\
\hline CTLD 8242-3 & CGRMV, CTLaV, CVA, LChV2, PDV, PNRSV & No & 46 \\
\hline CTLD 8242-3-Bi & CGRMV, CTLaV, CVA, LChV2, PDV, PNRSV & Yes & This study \\
\hline ARPD 95CI205R1 & CTLaV, CGRMV & No & 46 \\
\hline ARPD 95CI205R1-P2 & CTLaV, CVA & Yes & This study \\
\hline ARPD 95CI206 & CTLaV, CVA & Yes & 46 \\
\hline
\end{tabular}

a ACLSV = Apple chlorotic leafspot virus, CGRMV = Cherry green ring mottle virus, CNRMV = Cherry necrotic rusty mottle virus, CMLV = Cherry mottle leaf virus, $\mathrm{CRMaV}=$ Cherry rusty mottle-associated virus, $\mathrm{CTLaV}=$ Cherry twisted leaf-associated virus, CVA $=$ Cherry virus A, LChV2 $=$ Little cherry virus 2 , $\mathrm{PDV}=$ Prune $d$ warf virus, and PNRSV $=$ Prunus necrotic ringspot virus.

${ }^{b}$ Presence of CVA in source trees CRMD 98CI73R1 and CTLD 8265 was confirmed in this study using primer pair CVA fw1-rev1, which detects broader spectrum of CVA isolates (30).

c Source trees were propagated by grafting onto CVA-infected seedling rootstock. 
associated with viral replicase $(16,34,41)$ are also present in CRMaV, CTLaV, CNRMV, and CGRMV sequences.

ORFs 2, 3, and 4 encode proteins with $\mathrm{Mr}$ of 25, 12, and $7 \mathrm{kDa}$, respectively, and constitute the "potex-like" cell-to-cell movement-associated triple-gene block (TGB) proteins (32) present in some of members of the Alphaflexiviridae and Betaflexiviridae families.

The putative CP is encoded by ORF5 and consists of 269 aa for CRMaV, 268 aa for CGRMV, and 267 aa for both CNRMV and CTLaV. The predicted protein has a calculated $\mathrm{Mr}$ of $30 \mathrm{kDa}$, and contains the conserved core motif present in potexvirus and carlavirus CPs proposed to be involved in viral RNA-CP interaction (10).

The remaining two putative ORFs, ORF2a and ORF5a, potentially encode proteins of 119 and 157 aa with calculated Mr of 13 and $18 \mathrm{kDa}$, respectively. The existence of these putative ORFs is a unique feature found in this group of viruses but their expression has yet to be demonstrated.

Analysis of high-throughput sequencing. BLAST analysis of assembled contigs. Depending on the virus source tree, the total number of trimmed sequence reads ranged from 19 to 40 million. After removal of host sequences, de novo assembly of reads resulted in 6,500 to 17,000 contigs ranging from 97 to approximately $13,000 \mathrm{nt}$ in length that were subsequently used in BLASTN and BLASTX analyses against the nucleotide $\mathrm{nr} / \mathrm{nt}$ database of NCBI (Supplemental Table 3). Viruses identified by BLASTN were identical to that of the BLASTX results (Table 2) and all viruses identified from these two analyses were consistent with the previously determined virus profiles for each tree.

The proportion of sequence reads that were not assembled to contigs ranged from approximately 9 to $16 \%$, depending on the virus source tree. These reads contained low numbers of reads

TABLE 2. Number and length of longest assembled virus contigs, and the corresponding e-values of matching viruses in individual source tree by BLAST analysis $^{\mathrm{a}}$

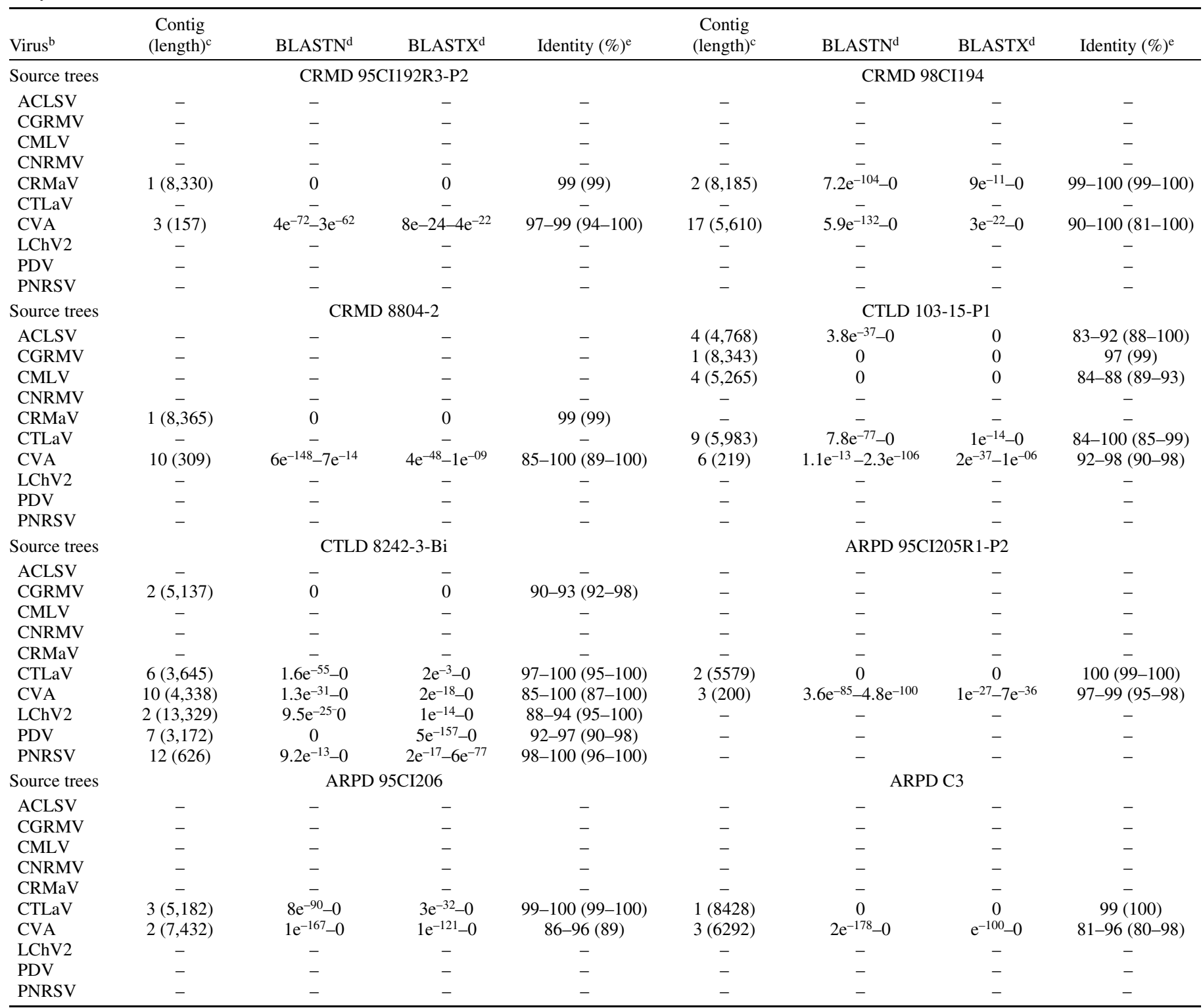

a Symbol: - indicates no corresponding virus contig.

${ }^{\mathrm{b}} \mathrm{ACLSV}=$ Apple chlorotic leafspot virus, CGRMV = Cherry green ring mottle virus, $\mathrm{CMLV}=$ Cherry mottle leaf virus, CNRMV $=$ Cherry necrotic rusty mottle virus, $\mathrm{CRMaV}=$ cherry rusty mottle-associated virus, $\mathrm{CTLaV}=$ cherry twisted leaf-associated virus, CVA $=$ Cherry virus A, LChV2 = Little cherry virus 2 , $\mathrm{PDV}=$ Prune dwarf virus, and PNRSV = Prunus necrotic ringspot virus.

${ }^{c}$ Numbers outside and inside the parentheses refer to the number and nucleotide length of longest assembled contig, respectively.

${ }^{\mathrm{d}}$ Ranges of BLASTX and BLASTN e-values of assembled virus contigs.

e Values outside and inside the parenthesis refer to percentage identity ranges of assembled virus contigs after BLASTN and BLASTX analyses, respectively. 
that mapped to the genome of viruses (Supplemental Table 4) whose identities, in general, were in agreement with the virus profile of each source tree. Very low numbers of reads $(\leq 27$ short reads) mapping to the genome of viruses not detected by BLASTN and BLASTX analyses were observed in some source trees. These included Apple chlorotic leafspot virus (ACLSV), CGRMV, and PDV in sources ARPD C3 and 95CI206, and $\mathrm{CRMaV}$ in APRD C3. However, examination of the detailed mapping profile of these reads in each source tree revealed poor coverage and depth (highest read coverage of 0.16 in ARPD C3 over the entire CGRMV genome). Moreover, specific RT-PCR tests for CGRMV and CRMaV (46) and ACLSV and PDV (48) in these source trees were negative. This suggests that the occurrence of these infrequent short virus-like sequence reads in ARPD 95CI206 and C3 was the result of spurious contamination. Taken together, the results of the BLAST analyses and mapping of unassembled reads indicate that no additional previously uncharacterized viruses are associated with CRMD, CTLD, or ARPD.

Mapping of reads to full genome sequences of CTLaV, CNRMV, CRMaV, and CGRMV. Sequence reads specific to CTLaV, CNRMV, CRMaV, and CGRMV in each of the source trees ranged from $0.1 \%$ of the total trimmed reads for CRMD 8804-2 (28,468 reads) to $5.5 \%$ for CRMD $98 \mathrm{CI} 194$ (1,460,098 reads). A large proportion (50 to $93 \%$ ) of these reads mapped to the corresponding virus isolate that was PCR amplified, cloned, and sequenced in each source tree. The same can be said of the proportion of reads covering the assembled full genomes of CTLaV C3 and CRMaV 8804 from high-throughput sequence reads of ARPD C3 and CRMD 8804-2 source trees, respectively. Because CTLaV 103-15 and CTLaV 101-13 share >99\% nt identity, reads from CTLD source 103-15-P1 that corresponded to CTLaV 101-13 and 103-15 (total of 245,527 reads) were remapped to each virus. Only 3,009 reads (1.2\%) remained unmapped. On the other hand, in total, 145,946 reads from CTLD 103-15-P1 and 107,652 reads for CTLD 8242-3-Bi were mapped to CGRMV, confirming the presence of this virus in these source trees.

Phylogenetic relationships. Comparisons between CTLaV, $C N R M V, C R M a V$, and CGRMV. Phylogenetic analyses of the replicase and $\mathrm{CP}$ amino acid sequences and whole-genome nucleotide sequences of members within the family Betaflexiviridae revealed four major clades (Figs. 1, 2, and 3), with each clade represented by the following viruses: CTLaV (clade I), CNRMV (clade II), CRMaV (clade III), and CGRMV (clade IV). Trees generated using neighbor-joining, as well as from sequences of other ORFs, yielded similar topologies (data not shown). The clustering into four distinct clades is in agreement with the recent
CP-based phylogeny for this family of viruses $(22,43)$, and the grouping of CTLD and ARPD virus sequences into the CTLaV clade corroborates the long-standing assertion of the close relationship between the causal viruses of CTLD and ARPD $(18,28)$.

The range of nucleotide and amino acid sequence identities between the replicase, CP (ORF5), and full genome of CTLaV, CNRMV, CRMaV, and CGRMV is presented in Table 3. The current species demarcation criteria for the family Betaflexiviridae for the whole replicase coding region suggests that viruses with $>72 \%$ nt or $>80 \%$ aa sequence identities should be regarded as strains of the same virus $(2,3)$. Following this recommendation, CTLaV, CNRMV, CRMaV, and CGRMV would be considered distinct viruses. However, this cut-off value for species demarcation would be incongruent for $\mathrm{CP}$ pairwise comparisons at both the nucleotide and amino acid level based on the current criteria $(2,3)$. This scenario is similar to the situation regarding Apricot latent virus (ApLV) and Apple stem pitting virus (ASPV) described by Youseff et al. (52), where priority was afforded the replicase gene identity values to resolve the taxonomic status of Cas12 and SB12452 isolates of ApLV. By giving preponderance to the replicase over the CP gene, CTLaV, CRMaV, CNRMV, and CGRMV would be grouped as distinct viruses within the family Betaflexiviridae. This supports the conclusion made previously for this group of viruses (46).

Comparison with other TGB coding viruses within members of the family Betaflexiviridae. The sequences determined for CTLaV, CNRMV, CRMaV, and CGRMV ultimately converged to form a cluster of sequences that is well diverged from fovea- and carlaviruses and from other TGB-coding unassigned viruses within the family Betaflexiviridae. This segregation of four sequence clades remained well separated in trees generated using the TGB-coding regions as well as in trees obtained by neighborjoining methods (data not shown). Comparison of amino acid identities of the proteins encoded across five ORFs also revealed considerably higher levels of identity between CTLaV, CNRMV, $\mathrm{CRMaV}$, and CGRMV than with foveaviruses and carlaviruses and other TGB-encoding unassigned viruses within the family Betaflexiviridae (Table 4).

\section{DISCUSSION}

The results presented in this study provide the first comprehensive analysis of full-length genomic sequences of CRMaV, CTLaV, CNRMV, and CGRMV using virus source trees that were previously characterized $(46,47)$. With the exception of CRMaV 8804 and CTLaV C3, whose full genomes were determined by high-throughput sequencing, the full genome of each virus was

TABLE 3. Coat protein, replicase, and full-genome sequence identity ranges of the four virus clades

\begin{tabular}{|c|c|c|c|c|c|c|c|c|c|c|c|c|}
\hline \multirow[b]{3}{*}{ Clade } & \multicolumn{12}{|c|}{ Range in percentage identities (average) ${ }^{\mathrm{a}}$} \\
\hline & \multicolumn{4}{|c|}{ Overall } & \multicolumn{4}{|c|}{ ORF1 (replicase) } & \multicolumn{4}{|c|}{ ORF5 (coat protein) } \\
\hline & $\begin{array}{c}\text { Clade I } \\
\text { (CTLaV) }\end{array}$ & $\begin{array}{l}\text { Clade II } \\
\text { (CNRMV) }\end{array}$ & $\begin{array}{l}\text { Clade III } \\
\text { (CRMaV) }\end{array}$ & $\begin{array}{l}\text { Clade IV } \\
\text { (CGRMV) }\end{array}$ & $\begin{array}{c}\text { Clade I } \\
\text { (CTLaV) }\end{array}$ & $\begin{array}{c}\text { Clade II } \\
\text { (CNRMV) }\end{array}$ & $\begin{array}{l}\text { Clade III } \\
\text { (CRMaV) }\end{array}$ & $\begin{array}{l}\text { Clade IV } \\
\text { (CGRMV) }\end{array}$ & $\begin{array}{c}\text { Clade I } \\
\text { (CTLaV) }\end{array}$ & $\begin{array}{c}\text { Clade II } \\
\text { (CNRMV) }\end{array}$ & $\begin{array}{l}\text { Clade III } \\
\text { (CRMaV) }\end{array}$ & $\begin{array}{l}\text { Clade IV } \\
\text { (CGRMV) }\end{array}$ \\
\hline $\begin{array}{l}\text { Clade I } \\
\text { (CTLaV) }\end{array}$ & $74-99$ & $\cdots$ & $\ldots$ & $\ldots$ & $\begin{array}{l}\text { 80-100 } \\
72-99\end{array}$ & $75-78$ & $73-75$ & $69-71$ & $\begin{array}{l}84-100 \\
81-100\end{array}$ & $77-82$ & $71-77$ & $69-75$ \\
\hline $\begin{array}{l}\text { Clade II } \\
\text { (CNRMV) }\end{array}$ & $70-72$ & $86-95$ & $\cdots$ & $\cdots$ & $69-71$ & $\begin{array}{l}91-96 \\
84-94\end{array}$ & $72-74$ & $68-69$ & $75-80$ & $\begin{array}{l}96-98 \\
90-97\end{array}$ & $77-80$ & $72-75$ \\
\hline $\begin{array}{l}\text { Clade III } \\
\text { (CRMaV) }\end{array}$ & $69-71$ & $68-69$ & $77-95$ & $\ldots$ & $68-70$ & $67-69$ & $\begin{array}{l}84-97 \\
75-94\end{array}$ & $70-71$ & $71-76$ & $73-76$ & $\begin{array}{l}\text { 89-100 } \\
86-97\end{array}$ & $70-74$ \\
\hline $\begin{array}{l}\text { Clade IV } \\
\text { (CGRMV) }\end{array}$ & $66-68$ & $65-66$ & $66-68$ & $82-95$ & $65-67$ & $64-65$ & $65-67$ & $\begin{array}{l}87-97 \\
80-95\end{array}$ & $69-74$ & $70-71$ & $70-75$ & $\begin{array}{l}96-99 \\
88-98\end{array}$ \\
\hline
\end{tabular}

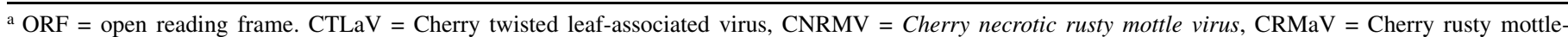
associated virus, and CGRMV = Cherry green ring mottle virus. For numbers in bold, the upper and lower values pertain to amino acid and nucleotide sequence identities, respectively between members within the clade. Values above and below numbers in bold correspond to amino acid and nucleotide percent sequence identities, respectively. 
determined through cloning and sequencing of full-genomelength RT-PCR amplicons. This approach was initially used in the full genome sequencing of Rupestris stem pitting-associated virus (D. E. V. Villamor, K. L. Druffel, and K. C. Eastwell, unpublished data), ASPV (4) and, more recently, two isolates of CRMaV 95CI192R3 and B48-C (44). The availability of full-length genomic clones of different isolates of CRMaV and CTLaV is a significant step in the development of infectious clones for each virus.
High-throughput sequencing has accelerated the rate at which pathogens associated with disease of unknown etiology are identified $(1,9,33)$. Because high-throughput sequencing does not require prior knowledge of the suspected pathogens in a diseased tree, this technique was pursued in order to determine whether previously uncharacterized viruses are associated with CRMD, CTLD, and ARPD. High-throughput sequencing did not reveal any signature virus sequences that could be ascribed to novel viruses, and mirrored the virus profiles determined by RT-PCR

\section{ORF1 (replicase)}

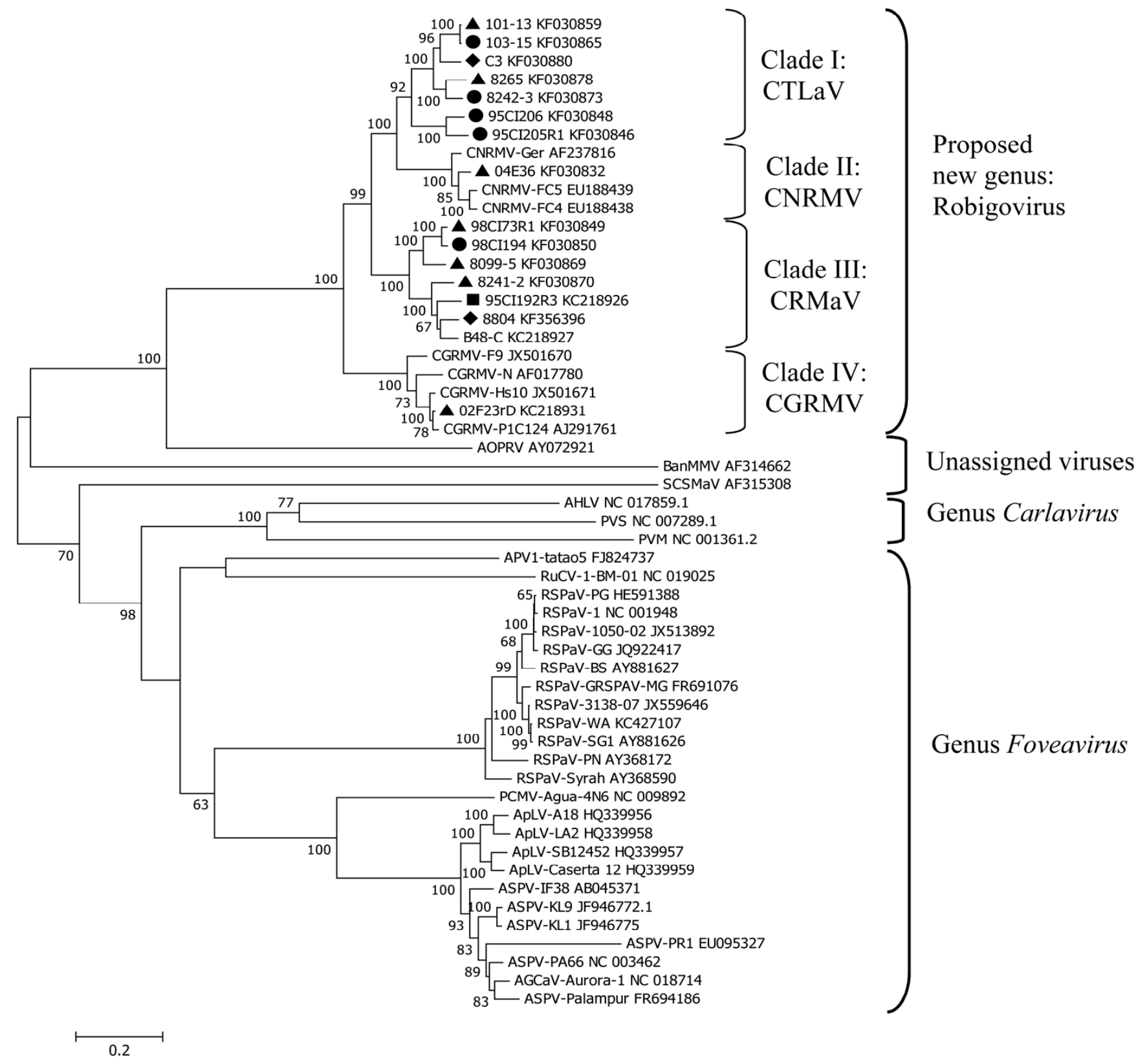

Fig. 1. Phylogenetic analysis of the amino acid sequence of the proteins encoded by replicase nucleotide sequences for Cherry twisted leaf-associated virus (CTLaV), Cherry necrotic rusty mottle virus (CNRMV), Cherry rusty mottle-associated virus (CRMaV), and Cherry green ring mottle virus (CGRMV). Tree was constructed using maximum likelihood with 1,000 bootstrap replicates of the MEGA program package, version 6. Branch nodes $>60 \%$ bootstrap values are shown. GenBank accession numbers are shown. Shaded symbols before each virus isolate name correspond to $\boldsymbol{\Delta}$, virus isolates whose full genomes were determined by reverse-transcription polymerase chain reaction (RT-PCR) but were not subjected to high-throughput sequencing; $\bullet$, virus isolates whose full genomes were determined by RT-PCR and were subjected to high-throughput sequencing; $\downarrow$, virus isolates whose full genomes were determined by high-throughput sequencing; and $\mathbf{\square}$, virus isolate whose full genome was determined previously (44) but was subjected to high-throughput sequencing. AgCaV = Apple green crinkleassociated virus, $\mathrm{AHLV}=$ American hop latent virus, $\mathrm{ApLV}=$ Apricot latent virus, $\mathrm{APV} 1=$ Asian prunus virus $1, \mathrm{AOPRV}=$ African oil palm ringspot virus, $\mathrm{ASPV}=$ Apple stem pitting virus, $\mathrm{BanMMV}=$ Banana mild mosaic virus, $\mathrm{BVX}=$ Banana virus $X, \mathrm{PCMV}=$ Peach chlorotic mottle virus, $\mathrm{PVM}=$ Potato virus $M$, $\mathrm{PVS}=$ Potato virus $S, \mathrm{RSPaV}=$ Rupestris stem pitting-associated virus, RuCV-1 = Rubus canadensis virus 1 , and $\mathrm{SCSMaV}=$ Sugarcane striate mosaicassociated virus. 
and ELISA analyses. Moreover, sequence reads mapping to the full genomic regions of $\mathrm{CRMaV}$ and $\mathrm{CTLaV}$ were recovered from CRMD and CTLD or ARPD source trees, respectively. It is worthwhile to note that all the CRMD and CTLD or ARPD source trees were also infected by CVA. Although CVA alone is considered latent in Prunus spp. $(11,23)$, its possible role in the etiologies of CRMD, CTLD, and ARPD needs to be examined further. Nevertheless, the associations of CRMaV with CRMD and CTLaV with CTLD or ARPD are robust (46), and the results presented in this study further support the causal relationship of these viruses with their respective diseases.

Aside from CGRMD, whose etiology is known to be definitely caused by CGRMV (53), the associations of CRMaV with CRMD, CTLaV with CTLD, and ARPD and CNRMV with CNRMD are supported by induction of distinct symptoms of each virus in biological woody indicators (46). The distinct disease symptoms associated with each of these viruses contribute to the proposed designation of these viruses as distinct species rather

\section{ORF5 (CP)}

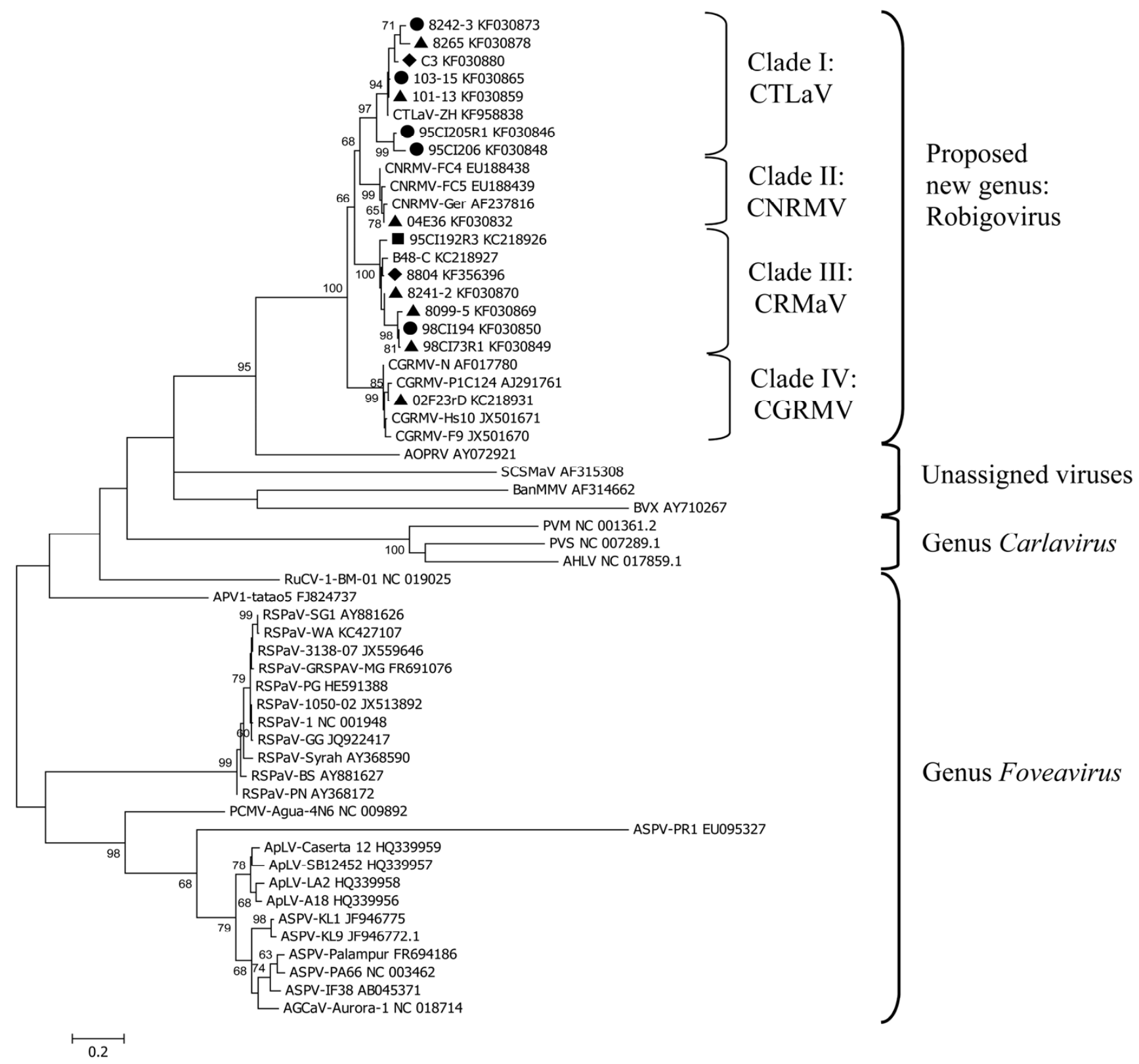

Fig. 2. Phylogenetic analysis of the amino acid sequence of the proteins encoded by coat protein nucleotide sequences for Cherry twisted leaf-associated virus (CTLaV), Cherry necrotic rusty mottle virus (CNRMV), Cherry rusty mottle-associated virus (CRMaV), and Cherry green ring mottle virus (CGRMV). Tree was constructed using maximum likelihood with 1,000 bootstrap replicates of the MEGA program package, version 6. Branch nodes $>60 \%$ bootstrap values are shown. GenBank accession numbers are shown. Shaded symbols before each virus isolate name correspond to $\boldsymbol{\Delta}$, virus isolates whose full genomes were determined by reverse-transcription polymerase chain reaction (RT-PCR) but were not subjected to high-throughput sequencing; $\bullet$, virus isolates whose full genomes were determined by RT-PCR and were subjected to high-throughput sequencing; $\downarrow$, virus isolates whose full genomes were determined by high-throughput sequencing; and $\mathbf{\square}$, virus isolate whose full genome was determined previously (44) but was subjected to high-throughput sequencing. AgCaV $=$ Apple green crinkleassociated virus, $\mathrm{AHLV}=$ American hop latent virus, $\mathrm{ApLV}=$ Apricot latent virus, $\mathrm{APV} 1=$ Asian prunus virus $1, \mathrm{AOPRV}=$ African oil palm ringspot virus, $\mathrm{ASPV}=$ Apple stem pitting virus, $\mathrm{BanMMV}=$ Banana mild mosaic virus, $\mathrm{BVX}=$ Banana virus $X, \mathrm{PCMV}=$ Peach chlorotic mottle virus, $\mathrm{PVM}=$ Potato virus $M$, $\mathrm{PVS}=$ Potato virus $S, \mathrm{RSPaV}=$ Rupestris stem pitting-associated virus, RuCV-1 = Rubus canadensis virus 1 , and $\mathrm{SCSMaV}=$ Sugarcane striate mosaicassociated virus. 


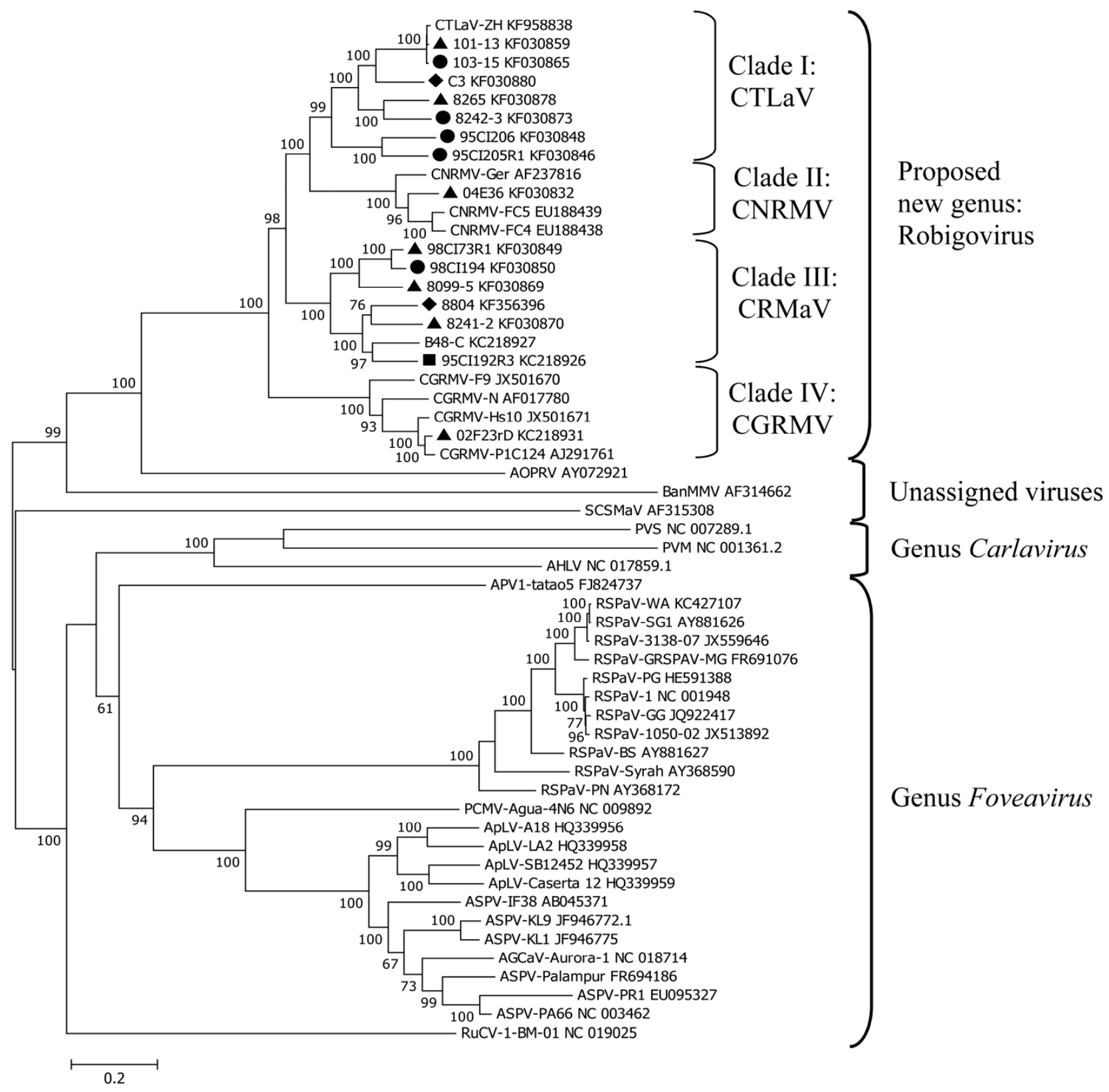

Fig. 3. Phylogenetic analysis of the amino acid sequence of the proteins encoded by whole-genome nucleotide sequences for Cherry twisted leaf-associated virus (CTLaV), Cherry necrotic rusty mottle virus (CNRMV), Cherry rusty mottle-associated virus (CRMaV), and Cherry green ring mottle virus (CGRMV). Tree was constructed using maximum likelihood with 1,000 bootstrap replicates of the MEGA program package, version 6. Branch nodes $>60 \%$ bootstrap values are shown. GenBank accession numbers are shown. Shaded symbols before each virus isolate name correspond to $\boldsymbol{\Delta}$, virus isolates whose full genomes were determined by reverse-transcription polymerase chain reaction (RT-PCR) but were not subjected to high-throughput sequencing; $\boldsymbol{\bullet}$, virus isolates whose full genomes were determined by RT-PCR and were subjected to high-throughput sequencing; $\$$, virus isolates whose full genomes were determined by high-throughput sequencing; and $\mathbf{\square}$, virus isolate whose full genome was determined previously (44) but was subjected to high-throughput sequencing. AgCaV = Apple green crinkleassociated virus, $\mathrm{AHLV}=$ American hop latent virus, $\mathrm{ApLV}=$ Apricot latent virus, $\mathrm{APV} 1=$ Asian prunus virus $1, \mathrm{AOPRV}=$ African oil palm ringspot virus, $\mathrm{ASPV}=$ Apple stem pitting virus, $\mathrm{BanMMV}=$ Banana mild mosaic virus, $\mathrm{BVX}=$ Banana virus $X, \mathrm{PCMV}=$ Peach chlorotic mottle virus, $\mathrm{PVM}=$ Potato virus $M$, $\mathrm{PVS}=$ Potato virus $S, \mathrm{RSPaV}=$ Rupestris stem pitting-associated virus, RuCV-1 = Rubus canadensis virus 1 , and $\mathrm{SCSMaV}=$ Sugarcane striate mosaicassociated virus.

than strains of a single virus species within the family Betaflexiviridae. The main criterion for inclusion of a virus in this family is the possession of a large potex-like replication protein (replicase) of greater than $195 \mathrm{kDa}(2,3)$. The results presented in this study revealed a replicase size of approximately $230 \mathrm{kDa}$ for CTLaV and CRMaV, providing concrete evidence for their inclusion within the family Betaflexiviridae.

The phylogenetic relationships of CTLaV, CNRMV, CRMaV, and CGRMV with members of the TGB-encoding genera as well as unassigned viruses reveal a need to reassess their current taxonomic classification status within the family Betaflexiviridae. Although the genome organizations of CTLaV, CNRMV, CRMaV, and CGRMV are similar to foveaviruses, which possess a replicase, TGB proteins, and $\mathrm{CP}$, they remain phylogenetically well separated from the foveaviruses across all ORFs. To merge CTLaV, CNRMV, CRMaV, and CGRMV as a different subgroup within the Foveavirus genus would not be in agreement with the phylogenetic clustering presented in this study. Moreover, com- 


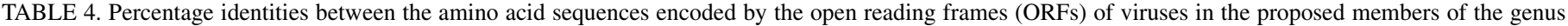

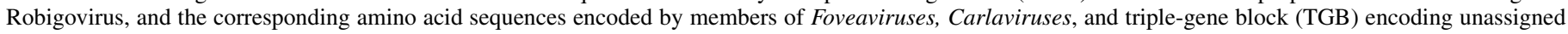
members within the family Betaflexiviridae

\begin{tabular}{|c|c|c|c|c|c|}
\hline Virus $^{a}$ & Replicase & TGB1 & TGB2 & TGB3 & Coat protein \\
\hline Foveaviruses & $26-36$ & $28-46$ & $24-40$ & $15-37$ & $15-31$ \\
\hline Carlaviruses & $30-33$ & $29-35$ & $29-41$ & $20-35$ & $21-35$ \\
\hline AOPRV & $41-43$ & $48-52$ & $35-41$ & $29-38$ & $38-41$ \\
\hline BanMMV & $29-30$ & $32-35$ & $36-40$ & $25-33$ & $25-28$ \\
\hline BVX & $\mathrm{n} / \mathrm{a}^{\mathrm{b}}$ & $28-34$ & $33-37$ & $18-24$ & $20-24$ \\
\hline SCSMaV & $29-31$ & $23-27$ & $22-30$ & $16-23$ & $19-25$ \\
\hline
\end{tabular}

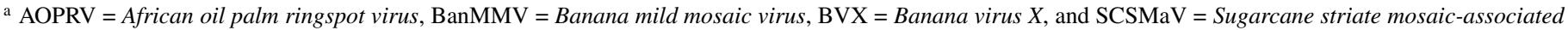
virus.

${ }^{\mathrm{b}}$ Full coding sequence of the ORF is not yet available.

parisons in the replicase and $\mathrm{CP}$ between foveaviruses and the CTLaV, CNRMV, CRMaV, and CGRMV cluster show amino acid identities below $40 \%$, values which usually indicate species belonging to different genera (2,3). An alternative is to group CTLaV, CNRMV, CRMaV, and CGRMV into a single genus. Whether other unassigned viruses within the family Betaflexiviridae could be included in this plausible genus can be addressed using the available sequence information, biology, and genome organization of these viruses. Excluding CTLaV, CNRMV, $\mathrm{CRMaV}$, and CGRMV, there are currently seven unassigned viruses within the family Betaflexiviridae. Three of these unclassified viruses (namely, Diuris virus A, Diuris virus B, and Hardenbergia virus $A$ ) do not encode for TGB proteins and, therefore, could reasonably be excluded in the plausible genus. The other four unassigned viruses possess TGB proteins and include the following viruses: African oil palm ringspot virus (AOPRV), Banana virus $X$, Banana mild mosaic virus, and Sugarcane striatemosaic-associated virus. Two possible taxonomic structures could be derived from this analysis. In one instance, a genus consisting of AOPRV and CTLaV, CNRMV, CRMaV, and CGRMV could be proposed based on the consistent clustering of AOPRV with CTLaV, CNRMV, CRMaV, and CGRMV in the CP, replicase, and whole-genome phylogenies as well as in the TGB proteins. In addition, only AOPRV showed $>40 \%$ aa identities in both the replicase and $\mathrm{CP}$ and relatively greater amino acid identities in the TGB proteins than with the fovea- and carlaviruses and the rest of the unassigned viruses within the family Betaflexiviridae. The inclusion of AOPRV in this possible genus would also prevent the diversity of the Foveavirus genus from getting even larger. To this possibility, CTLaV, CNRMV, CRMaV, and CGRMV would represent closely related viruses within the genus, with AOPRV being distantly related to them. The alternative structure would exclude AOPRV and suggest a separate genus consisting of only CTLaV, CNRMV, CRMaV, and CGRMV. Support for this structure relies on information available regarding the host range and genome organization of these viruses. The four viruses CTLaV, CNRMV, CRMaV, and CGRMV infect dicotyledonous, temperate fruit crops belonging to Prunus spp. whereas AOPRV is a tropical virus infecting monocotyledonous palms. Within the replicase ORF, an AlkB-like domain (31) is present in CTLaV, CNRMV, $\mathrm{CRMaV}$, and CGRMV, whereas this domain is absent in AOPRV. Although all these viruses possess TGB proteins, the three TGB ORFs in AOPRV overlap with each other, whereas only two ORFs (TGB2 and 3) overlap in CTLaV, CNRMV, CRMaV, and CGRMV. A valuable piece of information that can be used in the future to support this suggested taxonomic structure is the presence of nested putative ORFs ( $2 \mathrm{a}$ and $5 \mathrm{a}$ ) within TGB1 and 3 in CTLaV, CNRMV, CRMaV, and CGRMV. Although the expression of these nested ORFs has yet to be demonstrated, this feature is unique to CTLaV, CNRMV, CRMaV, and CGRMV.

Based on the arguments presented above, it seems appropriate to suggest a genus consisting of only CTLaV, CNRMV, CRMaV, and CGRMV. This is consistent with phylogenetic clustering and sequence comparisons but incorporates other important informa- tion on the biology and genome organization of these viruses. It is possible that AOPRV belongs to another genus yet to be created and evaluated when more virus species are characterized in the future. The name suggested for the proposed genus is Robigovirus (derived from the Latin word "robigo", meaning rust) and is chosen based on the symptoms associated with three viruses in sweet cherry cultivars. Of these three viruses, CRMaV and CNRMV are associated with rusty mottle symptoms, while CTLaV is associated with a milder and delayed expression of rusty mottle symptom (43).

\section{ACKNOWLEDGMENTS}

This work was supported, in part, by the Department of Plant Pathology, College of Agricultural, Human, and Natural Resource Sciences Agricultural Research Center Project Number WNP00754, Washington State University, Pullman 99164-6240; and by funding from the Washington State Tree Fruit Research Commission and the National Clean Plant Network of the United States Department of Agriculture Animal and Plant Health Inspection Service (USDA-APHIS). We thank M. Bernardy of the Canadian Plant Virus Collection, Agriculture and Agri-Food Canada, Summerland, BC, Canada, for providing material of ARPD source tree C3 under USDA-APHIS permit number P526P-11-02823.

\section{LITERATURE CITED}

1. Adams, I. P., Glover, R. H., Monger, W. A., Mumford, R., Jackeviciene, E., Navalinskiene, M., Samuitiene, M., and Boonham, N. 2009. Nextgeneration sequencing and metagenomic analysis: A universal diagnostic tool in plant virology. Mol. Plant. Pathol. 10:537-545.

2. Adams, M. J., Antoniw, J. F., Bar-Joseph, M., Brunt, A. A., Candresse, T., Foster, G. D., Martelli, G. P., Milne, R. G., and Fauquet, C. M. 2004. The new plant virus family Flexiviridae and assessment of molecular criteria for species demarcation. Arch. Virol. 149:1045-1060.

3. Adams, M. J., Candresse, T., Hammond, J., Kreuze, J. F., Martelli, G. P., Namba, S., Pearson, M. N., Ryu, K. H, Saldarelli, P., and Yoshikawa, N. 2012. Family Betaflexiviridae. Pages 920-941 in: Virus Taxonomy: Ninth Report of the International Committee on Taxonomy of Viruses. A. M. Q King, M. J. Adams, E. B. Carstens, and E. J. Lefkowitz, eds. Elsevier Academic Press, London.

4. Akinbade, S. A. 2012. Genetic diversity in viruses associated with apple green crinkle disease (AGCD). M.Sc. thesis, Washington State University, Pullman.

5. Al Rwahnih, M., Daubert, S., Golino, D., and Rowhani, A. 2009. Deep sequencing analysis of RNAs from a grapevine showing Syrah decline symptoms reveals a multiple virus infection that includes a novel virus. Virology 387:395-401.

6. Al Rwahnih, M., Daubert, S., Úrbez-Torres, J. R., Cordero, F., and Rowhani, A. 2011. Deep sequencing evidence from single grapevine plants reveals a virome dominated by mycoviruses. Arch. Virol. 156:397-403.

7. Al Rwahnih, M., Dave, A., Anderson, M. M., Rowhani, A., Uyemoto, J. K., and Sudarshana, M. R. 2013. Association of a DNA virus with grapevines affected by red blotch disease in California. Phytopathology 103: 1069-1076.

8. Al Rwahnih, M., Sudarshana, M. R., Uyemoto, J. K., and Rowhani A. 2012. Complete genome sequence of a novel vitivirus isolated from grapevine. J. Virol. 86:9545.

9. Cox-Foster, D. L., Conlan, S., Holmes, E. L., Palacios, G., Evans, J. D, Moran, N. A., Quan, P. L., Briese, T., Hornig, M., Geiser, D. M. 2007. A metagenomic survey of microbes in honey bee collapse disorder. Science 318:283-287. 
10. Dolja, V. V., Boyko, V. P., Agranovsky, A. A., and Koonin, E. V. 1991. Phylogeny of capsid proteins of rod-shaped and filamentous RNA plant viruses: Two families with distinct patterns of sequences and probably structure conservation. Virology 184:79-86.

11. Eastwell, K. C., and Bernardy, M. G. 1998. Relationship of cherry virus A to little cherry disease in British Columbia. Acta Hortic. 472:305-313.

12. Edgar, R. C. 2004. MUSCLE: Multiple sequence alignment with high accuracy and high throughput. Nucleic Acids Res. 32:1792-1797.

13. Elbeaino, T., Giampetruzzi, A., De Stradis, A., and Digiaro, M. 2014. Deep-sequencing analysis of an apricot tree with vein clearing symptoms reveals the presence of a novel betaflexivirus. Virus Res. 181:1-5.

14. Gentit, P., Foissac, X., Svanella-Dumas, L., Peypelut, M., Macquaire, G., and Candresse, T. 2002. Molecular characterization of foveaviruses associated with cherry necrotic mottle leaf disease and complete sequencing of an European isolate of Cherry green ring mottle virus. Arch. Virol. 147:1033-1042.

15. Giampetruzzi, A., Roumi, V., Roberto, R., Malossini, U., Yoshikawa, N., La Notte, P., Terlizzi, F., Credi, R., and Saldarelli, P. 2012. A new grapevine virus discovered by deep sequencing of virus- and viroidderived small RNAs in cv. Pinot gris. Virus Res. 163:262-268.

16. Gorbalenya, A. E., Blinov, V. M., Donchenko, A. P., and Koonin, E. V. 1989. An NTP-binding motif is the most conserved sequence in a highly diverged monophyletic group of proteins involved in positive strand RNA viral replication. J. Mol. Biol. 28:256-268.

17. Hall, T. A. 1999. BioEdit: A user-friendly biological sequence alignment editor and analysis program for Windows 95/98/NT. Nucleic Acids Symp. Ser. 41:95-98.

18. Hansen, A. J., Parish, C. L., and Pine, S. T. 1976. Apricot ring pox. Pages 45-49 in: Virus Diseases and Noninfectious Disorders of Stone Fruits in North America. R. M. Gilmer, J. D. Moore, G. Nyland, M. F. Welsh, and T. S. Pine, eds. U.S. Dep. Agric. Agric. Handb. 437.

19. James, D. 1992. Isolation of a high molecular weight dsRNA associated with twisted leaf disease in cherry. Can. J. Plant Pathol. 14:281-284.

20. James, D. 2011. Cherry twisted leaf disease and its associated viruses. Pages 143-146 in: Virus and Virus-like Diseases of Pome and Stone Fruits. A. Hadidi, M. Barba, T. Candresse, and W. Jelkmann, eds. American Phytopathological Society, St. Paul, MN.

21. James, D., Howell, W. E., and Martin, R. R. 1995. Identification of a flexeous virus associated with cherry twisted leaf disease. Acta Hortic. 386:86-91.

22. James, D., Varga, A., and Lye, D. 2014. Analysis of the complete genome of a virus associated with twisted leaf disease of cherry reveals evidence of a close relationship to unassigned viruses in the family Betaflexiviridae. Arch. Virol. 159:2463-2468.

23. Jelkmann, W. 1995. Cherry virus A: cDNA cloning of dsRNA, nucleotide sequence analysis and serology reveal a new plant capillovirus in sweet cherry. J. Gen. Virol. 76:2015-2024.

24. Li, L., Pesavento, P. A., Shan, T., Leutenegger, C. M., Wang, C., and Delwart, E. 2011. Viruses in diarrhoeic dogs include novel kobuviruses and sapoviruses. J. Gen. Virol. 92:2534-2541.

25. Li, R., and Mock, R. 2008. Characterization of a flowering cherry strain of Cherry necrotic rusty mottle virus. Arch. Virol. 153:973-978.

26. Liberti, D., Marais, A., Svanella-Dumas, A., Ragozzino, A., and Candresse, T. 2005. Partial genome sequence of an apricot isolate of Cherry green ring mottle virus. Arch. Virol. 150:185-188.

27. Lott, T. B. 1945. "Lambert mottle" a transmissible disease of sweet cherry. Sci. Agric. 27:260-262.

28. Lott, T. B., and Keane, F. W. L. 1960. The association of the virus disease twisted leaf of cherry and ring pox of apricot. Plant Dis. Rep. 44:243-245.

29. Makarova, K. S., Aravind, L., and Koonin, E. V. 2000. A novel superfamily of predicted cysteine proteases from eukaryotes, viruses and Chlamydia pneumoniae. Trends Biochem. Sci. 25:50-52.

30. Marais, A., Svanella-Dumas, L., Barone, M., Gentit, P., Faure, C., Charlot, G., Ragozzino, A., and Candresse, T. 2012. Development of a polyvalent RT-PCR detection assay covering the genetic diversity of Cherry capillovirus A. Plant Pathol. 61:195-204.

31. Martelli, G.P., Adams, M. J., Kreuze, J. F., and Dolja, V. V. 2007. Family Flexiviridae: A case study in virion and genome plasticity. Annu. Rev. Phytopathol. 45:73-100.

32. Morozov, S. Y., and Solovyev, A. G. 2003. Triple gene block: Modular design of a multifunctional machine for plant virus movement. J. Gen. Virol. 84:1351-1366.

33. Palacios, G., Druce, J., Du, L., Tran, T., Birch, C., Briese, T., Conlan, S.,
Quan, P. L., Hui, J., Marshall, J., Simons, J. F., Egholm, M., Paddock, C. D., Shieh, W. J., Goldsmith, C. S., Zaki, S. R., Catton, M., and Lipkin, W. I.. 2008. A new arenavirus in a cluster of fatal transplant-associated diseases. New Engl. J. Med. 358:991-998.

34. Poch, O., Sauvaget, I., Delarue, M., and Tordo, N. 1989. Identification of four conserved motifs among the RNA-dependent polymerase encoding elements. EMBO J. 8:3867-3874.

35. Poojari, S., Alabi, O. J., Fofanov, V. Y., and Naidu, R. N. 2013. A leafhopper-transmissible DNA virus with a novel evolutionary lineage in the family geminiviridae implicated in grapevine redleaf disease by nextgeneration sequencing. PLoS One 8:e64194. doi:10.1371/journal. pone.0064194

36. Posnette, A., and Cropley, R. 1957. A canker disease of cherry trees caused by virus infection. Plant Pathol. 6:85-87.

37. Reeves, E. L. 1940. Rusty mottle, a new virosis of cherry. (Abstr.) Phytopathology (Abstr.) 30:789

38. Reeves, E. L., and Richards, B. L. 1946. A rusty mottle-like virus disease of the sweet cherry in Utah. (Abstr.) Phytopathology 36:409.

39. Rhoads, A. 1945. Symptom expression of rusty mottle in Utah sweet cherry orchards. Plant Dis. Rep. 29:613-614.

40. Rott, M. E., and Jelkmann, W. 2001. Complete nucleotide sequence of cherry necrotic rusty mottle virus. Arch. Virol. 146:395-401.

41. Rozanov, M. N., Koonin, E. V., and Gorbalenya, A. E. 1992. Conservation of the putative methyltransferase domain: A hallmark of the "Sindis-like" supergroup of positive-strand RNA viruses. J. Gen. Virol. 73:2129-2134.

42. Sabanadzovic, S., Ghanem-Sabanadzovic, N. A., and Gorbalenya, A. E. 2009. Permutation of the active site of putative RNA-dependent RNA polymerase in a newly identified species of plant alpha-like virus. Virology 39:1-7.

43. Stout, G. L. 1949. Cherry bark blister. Calif. Dep. Agric. Bull. 38:257-260.

44. Tamura, K., Stecher, G., Peterson, D., Filipski, A., and Kumar, S. 2013. MEGA6: Molecular Evolutionary Genetics Analysis Version 6.0. Mol. Bio. Evol. 30:2725-2729.

45. Trojnar, E., Sachsenroder, J., Twardziok, S., Reetz, J., Otto, P. H., and Johne, R. 2013. Identification of an avian group A rotavirus containing a novel VP4 gene with a close relationship to those of mammalian rotaviruses. J. Gen. Virol. 94:136-142.

46. Villamor, D. E. V., and Eastwell, K. C. 2013. Viruses associated with rusty mottle and twisted leaf diseases of sweet cherry are distinct species. Phytopathology 103:1287-1295.

47. Villamor, D. E. V., Ward, K. F., Collman, S. J., and Eastwell, K. C. 2013. First report of infection of cherry rusty mottle associated virus in Portuguese laurel (Prunus lusitanica) in Washington State. Plant Dis. 98:699.

48. Villamor, D. V., Druffel, K. L., and Eastwell, K. C. 2013. Complete nucleotide sequence of a virus associated with rusty mottle disease of sweet cherry (Prunus avium). Arch. Virol. 158:1805-1810.

49. Vives, M. C., Velazquez, K., Pina, J. A., Moreno, P., Guerri, J., and Navarro, L. 2013. Identification of a new enamovirus associated with citrus vein enation disease by deep sequencing of small RNAs. Phytopathology 103:1077-1086

50. Wadley, B. N., and Nyland, G. 1976. Rusty mottle group. Pages 242-249 in: Virus Diseases and Noninfectious Disorders of Stone Fruits in North America. R. M. Gilmer, J. D. Moore, G. Nyland, M. F. Welsh, and T. S. Pine, eds. U.S. Dep. Agric. Agric. Handb. 437.

51. Wang, W., Jiang, D., Niu, F., Lu, M., Wang, H., and Li, S. 2013. Complete nucleotide sequences of two isolates of Cherry green ring mottle virus from peach (Prunus persica) in China. Arch. Virol. 158:707-710.

52. Youssef, F., Marais, A., Faure, C., Barone, M., Gentit, P., and Candresse, T. 2011. Characterization of Prunus-infecting Apricot latent virus-like Foveaviruses: Evolutionary and taxonomic implications. Virus Res. 155:440-445.

53. Zagula, K. R., Aref, N. M., and Ramsdell, D. C. 1989. Purification, serology, and some properties of a mechanically transmissible virus associated with green ring mottle disease in peach and cherry. Phytopathology 79:451-456.

54. Zhang, Y. P., Kirkpatrick, B. C., Smart, C. D., and Uyemoto, J. K. 1998. cDNA cloning and molecular characterization of cherry green ring mottle virus. J. Gen. Virol. 79:2275-2281.

55. Zhang, Y. P., Mink, G. I., Tiffany, M. G., and Howell, W. E. 1992. Isolation of viruses associated with cherry twisted leaf, apricot ringpox, and apricot pit pox diseases and their relationship to apple stem pitting virus. Phytopathology 82:1149. 